\title{
Vibration-induced jamming transition in granular media
}

\author{
G. D'Anna and G. Gremaud \\ Institut de Génie Atomique, Département de Physique, Ecole Polytechnique Fédérale de Lausanne, CH-1015 Lausanne, \\ Switzerland
}

(November 15, 2018)

\begin{abstract}
The quasi-static frequency response of a granular medium is measured by a forced torsion oscillator method, with forcing frequency $f_{p}$ in the range $10^{-4} \mathrm{~Hz}$ to $5 \mathrm{~Hz}$, while weak vibrations at highfrequency $f_{s}$, in the range $50 \mathrm{~Hz}$ to $200 \mathrm{~Hz}$, are generated by an external shaker. The intensity of vibration, $\Gamma$, is below the fluidization limit. A loss factor peak is observed in the oscillator response as a function of $\Gamma$ or $f_{p}$. In a plot of $\ln f_{p}$ against $1 / \Gamma$, the position of the peak follows an Arrheniuslike behaviour over four orders of magnitude in $f_{p}$. The data can be described as a stochastic hopping process involving a probability factor $\exp \left(-\Gamma_{j} / \Gamma\right)$ with $\Gamma_{j}$ a $f_{s}$-dependent characteristic vibration intensity. A $f_{s}$-independent description is given by $\exp \left(-\tau_{j} / \tau\right)$, with $\tau_{j}$ an intrinsic characteristic time, and $\tau=\Gamma^{n} / 2 \pi f_{s}, \mathrm{n}=0.5-0.6$, an empirical control parameter with unit of time. $\tau$ is seen as the effective average time during which the perturbed grains can undergo structural rearrangement. The loss factor peak appears as a crossover in the dynamic behaviour of the vibrated granular system, which, at the time-scale $1 / f_{p}$, is solid-like at low $\Gamma$, and the oscillator is jammed into the granular material, and is fluid-like at high $\Gamma$, where the oscillator can slide viscously.
\end{abstract}

\section{INTRODUCTION}

In a granular medium at rest the grains can be disposed in an enormous number of different configurations. A weak external disturbance, but powerful enough to overcome locally the friction force between two grains, allows the granular systems to rearrange and to switch between these "blocked" configurations. The macroscopic behavior of a weakly disturbed granular medium is, therefore, essentially controlled by statistical properties of such transitions. It is of great interest to study this kind of problem, since it may be a prototype of slow dynamics behaviors observed in other physical systems [1]. The slow dynamics of weakly disturbed granular media has been evidenced by the classical compaction experiments of Knight et al. [2] Another approach is the experiment of Albert et al. [3], in which a large solid object is pulled slowly through a granular medium. The motion of the object appears as resisted by chains of jammed particles [4] [5], which support compressive stress. Beyond an elastic regime at very small pulling force, the macroscopic motion of the object is a succession of stick-slip events, where compressive stress is continuously built up in particle chains, and abruptly released. At these successive unjamming events the system switches between blocked configurations.

In this paper we implement a novel experimental method to study the problem: we exploit a forced torsion oscillator [6] immersed in the granular medium, and in presence of external weak vibration, as shown in Fig. 1. We use a dynamic method, which provides more information than the simple increase toward the unjamming threshold. In fact, the amplitude of the angular displacement of the oscillator increases sharply when the unjamming threshold forcing torque amplitude is approached, and at the same time the angular displacement lags behind the sinusoidal torque. This "phase lag" is determined by an energy dissipation which occurs in the granular medium when grains start to slip one against the other. The dynamic method gives access to both elastic and dissipation parameters of the granular material during the slow dynamics.

\section{EXPERIMENTAL}

In the experiment, we hold a granular medium at a given high-frequency vibration intensity, quantified by the normalized acceleration $\Gamma=a_{s} \omega_{s}^{2} / g$, with $a_{s}$ and $f_{s}=\omega_{s} / 2 \pi$ the amplitude and frequency of the vertical sinusoidal vibration, $g$ the acceleration of gravity. At the same time, we measure the complex frequency response, $G$, of the granular medium (or the susceptibility $\chi=G^{-1}$ ) by a low-frequency forced torsion oscillator [6], at the forcing frequency $f_{p}=\omega_{p} / 2 \pi$, with $f_{p} \ll f_{s}$. In the oscillator method (see Fig. 1), the rotating probe of the oscillator is immersed at a depth $L$ into a large metallic bucket (height $96 \mathrm{~mm}$, diameter $94 \mathrm{~mm}$ ) filled with glass beads of diameter $d=1.1 \pm 0.05 \mathrm{~mm}$ with smoothly polished surfaces. The probe is covered by a layer of beads, glued on by an epoxy, and its effective radius is $R_{e}$. All data presented here are obtained with $L=20 \mathrm{~mm}$ and $R_{e} \approx 2 \mathrm{~mm}$. We perform dynamic experiments: the oscillator is forced into torsion oscillation by a torque $T(t)=T_{o} \exp \left(i \omega_{p} t\right)$ of frequency in the range $10^{-4} \mathrm{~Hz}$ to $5 \mathrm{~Hz}$, and the angular displacement, $\theta(t)$, is optically detected. An analyzer measures the complex frequency response of the oscillator, given by $G=T / \theta$. Typically we record the $\operatorname{argument}, \arg \left(G_{1}\right)$, and the absolute modulus, $\left|G_{1}\right|$, of the first harmonic, as a function of either $T_{0}, \Gamma$, or $f_{p}$. We report the quantity $\tan \left[\arg \left(G_{1}\right)\right]$, 
which for a linear system coincides with the loss factor. The oscillator, when not immersed, can be assumed elastic, with $T=G_{p} \theta$, where $G_{p}=18 \times 10^{-3} \mathrm{~N} \mathrm{~m} / \mathrm{rad}$ is the torsion constant of the suspension wires. Notice that in this work the maximum displacement of a point at the surface of the $2 \mathrm{~mm}$ probe is of the order of $0.1 \mathrm{~mm}$, i.e., much smaller than the glass bead diameter.

An accelerometer provides a precise measurement of $\Gamma$, which can be varied from $2 \times 10^{-3}$ to above 1 . The minimum value of $\Gamma$ is limited by the accelerometer sensitivity. We vary $\Gamma$ by changing $a_{s}$ at fixed $f_{s}$, while $f_{s}$ is selected in the range $50 \mathrm{~Hz}$ to $200 \mathrm{~Hz}$. The whole system is placed on an anti-seismic table. Moisture-induced ageing effects [7] [6], and interstitial gas effects [8], are not observed for the large bead size used here, and measurements are performed at uncontrolled ambient air. In order to control compaction effects [2], all measurements are taken in the same conditions, e.g., starting from a granular material shaken at high $\Gamma$ and low $f_{s}$ for several minutes. Compaction effects are apparently negligible in the time scale of the experiments for $f_{p}>0.01 \mathrm{~Hz}$, but may be present in the data at very-low frequency.

\section{RESULTS}

A typical experimental result is reported in Fig. 2, which shows $\tan \left[\arg \left(G_{1}\right)\right]$ and $\left|G_{1}\right|$, measured as a function of the amplitude of the applied torque $T_{0}$, for different $\Gamma$. With the vibrator off, that is for $\Gamma<2 \times 10^{-3}$, the response is similar to the one reported previously [6], with a typical loss factor peak at a torque denoted $T_{0}^{*}$ and a modulus step between two levels denoted $G_{j a m}$ and $G_{p}$. The dependence of the loss peak on the geometrical parameters of the experiment is summarized [6] by the empirical relation $T_{0}^{*} \propto \mu_{s} L^{2} R_{e}^{2}$, with $\mu_{s}$ the coefficient of static friction between the glass beads. In this "zero temperature-like" conditions, the loss factor peak can be easily explained: at very low applied torque, $T_{0} \ll T_{0}^{*}$, the oscillator probe is jammed into the granular material, and only elastic deformations arise, resulting in a purely elastic dynamic response, with a negligible loss factor and a constant absolute modulus $G_{j a m}$. By increasing the torque amplitude, the oscillator probe unjams as the local force between a pair of glass beads somewhere in the medium becomes large enough for the two beads to slip one against the other, dissipating energy by solid friction. The maximum ratio of dissipated over furnished energy, that is a maximum of the loss factor, arises at $T_{0}^{*}$, which can be seen as the average torque at which the oscillator probe unjams. At high torque amplitude, $T_{0} \gg T_{0}^{*}$, the oscillator slides almost freely into the granular medium, and the absolute modulus tends to the torsion constant of the suspension wires, $G_{p}$.

With the vibrator on, one expects that the external vibration facilitates the unjamming of the oscillator. By increasing $\Gamma$, the modulus $\left|G_{1}\right|$ decreases monotonically, while $\tan \left[\arg \left(G_{1}\right)\right]$ first increases and then decreases, going through a maximum, as clearly visible in Fig. 2. The fact that the external vibration drives the system through a maximum in the loss factor is evidence that the vibration-induced fluctuations can unjam the oscillator probe. Moreover, below $T_{0}^{*}$ the response is essentially independent of $T_{0}$, i.e., there is a linear regime. The linearity is confirmed also by a negligible high harmonics signal (not shown) for all $\Gamma$.

The general behavior in the linear regime is better rendered by Fig. 3 which shows the previous maximum in the loss factor as characteristic "jamming" peaks observed as a function of $\Gamma$ for various forcing frequencies $f_{p}$. In Fig. 3 we obtain that for the low-torque amplitude, selected in the linear regime, i.e., $T_{0} \ll T_{0}^{*}$, and at low $\Gamma$, the applied torque alone is unable to unjam the oscillator probe and the response is elastic. However, by increasing $\Gamma$, unjamming is induced by the external vibration, and the response displays a loss factor peak. The data shown in Fig. 3 are collected by decreasing $\Gamma$, but no difference is observed in following runs if $\Gamma$ is successively increased, decreased and so on, as shown in Fig. 4 for one of the curves of Fig. 3. We say that the response is "reversible", although at a mesoscopic level energy is continuously dissipated. The loss factor peak can be seen as the crossover between two different behaviours in the dynamics of the vibrated granular system: at the time-scale set on by the forced oscillator, i.e., $1 / f_{p}$, the granular system appears solid-like at low- $\Gamma$, while it appears fluid-like at high- $\Gamma$. It is a kind of glass, or jamming transition [20], where the oscillator gets stuck in the glassy granular medium.

Of course, since the "jamming" peak in Fig. 3 shifts with $f_{s}$, the same peak can be observed as a function of the forcing frequency $f_{p}$, as shown in Fig. 5, for various $\Gamma$. At high forcing frequency (but still $f_{p} \ll f_{s}$ ), the applied torque alone is unable to unjam the oscillator probe and the response is elastic, with negligible loss factor and modulus $G_{j a m}$. However, by decreasing $f_{p}$, i.e., by increasing the time scale of the probing oscillator, the response evolves toward the one of the unjammed oscillator, with modulus $G_{p}$.

From the shift of the previous "jamming" peaks with $f_{p}$ or $\Gamma$, an Arrhenius-like semilogarithmic plot can be obtained, as shown in Fig. 6. The data points, for a given $f_{s}$, obey an exponential behavior over four decades in frequency. This is strong evidence for the underlying unjamming process to be a statistical, activated-like hopping process and that some of the usual statistical concepts of thermal systems can be extended to a vibrated granular material. A first, obvious approach consists of formally writing the rate of the hopping process as $R=\nu_{0} \exp \left(-\Gamma_{j} / \Gamma\right)$, with $\Gamma_{j}$ a characteristic normalized acceleration at which unjamming occurs, $\nu_{0}$ an attempt frequency, and $\Gamma$ the vibration intensity, playing the role of a temperature-like parameter. In the linear regime, 
a peak in the loss factor is expected to arise when the forcing frequency matches the hopping rate, i.e., when $\omega_{p}=R$, and $\Gamma_{j}$ appears as the "slope" of a straight line in a plot of $\ln R$ against $1 / \Gamma$; however, then $\Gamma_{j}$ depends on $f_{s}$ (see Fig. 6 ), which means that $\Gamma_{j}$ is not an intrinsic property of the granular material.

To overcome this difficulty, we search a "scaling" of $\Gamma$ and $f_{s}$ which eliminates the $f_{s}$ dependence. We find that as a function of the inverse of $\Gamma^{n} / \omega_{s}$, with $n=0.5$, the data for different $f_{s}$ have almost the same "slope", as shown in Fig. 7. Hence, we write the hopping probability per unit time as $R=\nu_{0} \exp \left(-\tau_{j} / \tau\right)$, with $\tau_{j}$ a characteristic time of the unjamming process, and $\tau=\Gamma^{1 / 2} / \omega_{s}$ a parameter which has the unit of time [9]. (Fitting values are given in Fig. 7) Alternatively (Fig. 8), we find also that a "scaling" of the form $\tau=\Gamma^{n} / \omega_{s}$, with $n=0.57$, almost collapses the data of Fig. 6 over the same straight line. This empirical definition is appealing since both the parameters $\tau$ and $\tau_{j}$ we introduce, are independent of the vibration frequency $f_{s}$. The unjamming time $\tau_{j}$ experimentally is an intrinsic parameter of the granular system, which for a given $L$ and $R_{e}$ is likely to depend on "mesoscopic" parameters such as the grain size and shape, and on "microscopic" parameters controlling the nature of the contact forces between grains. For $n=0.5$ the externally controlled parameter $\tau$ appears as a typical time of the vibrated granular system in the gravitational field: $\Gamma^{1 / 2} / \omega_{s}=\left(a_{s} / g\right)^{1 / 2}$ is the time of flight of a body, initially at rest, falling for a distance $a_{s}$. (It is also the period of a simple oscillator with thread $a_{s}$ and freely swinging.) Notice that $\tau$ is not a kinetic energy-type temperature, as defined for vigorously vibrated gas-like granular phases; indeed, for weakly excited granular systems, configurational statistics on slow degrees of freedom can be decoupled from fast kinetic aspects [1] [10] 111 12. 13. 14.

\section{DISCUSSION}

That the rate of our "activated-like" process is better given by a probability factor involving the ratio of characteristic times, $\exp \left(-\tau_{j} / \tau\right)$, and not, e.g., by the ratio of vibration intensities, $\exp \left(-\Gamma_{j} / \Gamma\right)$, is not surprising since the fundamental phenomenon is the rate of energy dissipation. What is the mesoscopic nature of the hopping processes at the length scale of the glass beads? Since we observe an elastic regime at low $\Gamma$, we conclude that the oscillator probe is completely jammed and no dissipative events (i.e., no slipping events) arise. We can suppose the system to oscillate elastically around one unique blocked configuration. In the picture, below the loss factor peak, the external high-frequency vibration propagates in the system as elastic fluctuations only. Such elastic fluctuations are non-dissipative vibrations of the force-chain network which holds the oscillator probe in place. By in- creasing $\Gamma$, a large number of increasingly energetic elastic fluctuations arise (or increasingly longer if we focus on the parameter $\left.\tau=\Gamma^{n} / \omega_{s}\right)$, and the force-chain network can be seen as exploring different elastic configurations until a critical configuration is reached. A critical configuration is such that, somewhere, the local friction force between two glass beads is overcome and slipping arises, momentarily unjamming the oscillator probe. The system can switch to another blocked configuration.

A single slipping event possibly triggers a large-scale non-elastic rearrangement of the beads, that is an internal micro-avalanche involving two or more beads. (We emphasize that a massive object moving into a granular medium can introduce local fluidization. The inertia acquired by the object during a slip may be large enough to overcome the resisting force of the granular medium, and the object moves further by successive failure, or "inertial" fluidisation, of the resisting grains arrangement. This effect can be reduced by immersing the object deep enough, so that the resisting force is larger than the inertial force.) Afterwards, the oscillator probe gets jammed by a new force-chain network in a slightly different position. The macroscopic slow rotation of the oscillator is a sequence of stick-slips, where large fluctuations causing a slip are rare events if compared to the numerous elastic fluctuations. Hence, the dynamics is controlled by the extreme fluctuations in the force-chain network, even if elastic, or under-critical fluctuations occur in much larger number. Since a slipping event involves inelastic microscopic processes at the interface between grains, such as plastic and viscoplastic deformation, fatigue, surface fracture, blow out of capillary bridges, and other forms of localized dissipative processes [15], a slipping event requires a minimum finite time to occur. Let this time be $\tau_{j}$. The "thermal" time $\tau$ can be seen as the average time-window during which the grains have some freedom to rearrange their position and, possibly, reach a critical slipping configuration and unjam. As a consequence, unjamming is determined by the occurrence probability of a window time $\tau_{a}$ greater as, or equal to $\tau_{j}$. Even though we do not know the probability distribution of $\tau_{a}$, according to extreme order statistics theory [16] 17] [18] [19], we can speculate the occurrence probability of unjamming events to be $\exp \left(-\tau_{j} / \tau\right)$. This gives the rate of the extreme fluctuations, namely $R=\nu_{0} \exp \left(-\tau_{j} / \tau\right)$.

In summarizing, we observe a peak in the loss factor as a function of the empirical control parameters $\tau$ or $\Gamma$. The peak can be viewed as a crossover, at the time-scale $1 / f_{p}$ set on by the forced oscillator, in the dynamics of the vibrated granular medium: such a crossover separates a "low-temperature" (short $\tau$, or small $\Gamma$ ) solid-like behaviour where the oscillator probe is jammed in the granular medium, from a "high-temperature" (long $\tau$, or large $\Gamma$ ) fluid-like dynamic behaviour. This crossover follows an Arrhenius-like form in the $\ln f$ vs. $1 / \Gamma$ plane, reminiscent of the mechanical response of usual glass- 
froming materials.

[1] A. Barrat, J. Kurchan, V. Loreto, and M. Sellitto, Phys. Rev. Lett. 85, 5034 (2000).

[2] J. B. Knight, C. G. Fandrich, C. N.Lau, H. M. Jaeger, and S. R. Nagel, Phys. Rev. E 51, 3957 (1995).

[3] I. Albert, et al., Phys. Rev. Lett. 84, 5122 (2000).

[4] M. E. Cates, J. P. Wittmer, J.-P. Bouchaud, and P. Claudin, Phys. Rev. Lett. 81, 1841 (1998).

[5] A. J. Liu, and S. R. Nagel, Nature 396, 21 (1998).

[6] G. D'Anna, Phys. Rev. E 62, 982 (2000).

[7] L. Bocquet, E. Charlaix, S. Ciliberto,. and J. Crassous, Nature 396, 735 (1998).

[8] H. K. Pak, E. Van Doorn, and R. P Behringer, Phys. Rev. Lett. 74, 4643 (1995).

[9] When the vibration frequency is not a control parameter, one can write the "activated" behavior in term of probability factors involving $\Gamma$ instead than $\Gamma^{n} / \omega_{s}$. As shown in Fig. 6, for a fixed vibration frequency $f_{s}$, formally writing the rate of the activated-like hopping process as $R=\nu_{0} \exp \left(-\Gamma_{j} / \Gamma\right)$, suffices.

[10] S. F. Edwards, in: Granular Matter, (ed A. Mehta.) 121140 (Springer-Verlag, New York 1994).

[11] E. Clement, and J. Rajchenbach, Europhys. Lett. 16, 133 (1991).

[12] L. Berthier, L. F. Cugliandolo, and J. L. Iguain, Phys. Rev. E 63, 51302 (2001).

[13] M. Nicodemi, and A. Coniglio, Phys. Rev. Lett. 82, 916 (1999).

[14] A. Mehta, and G. C. Barker, J. Phys.: Condens. Matter 12, 6619 (2000).

[15] P. P. Bowden, and D. Tabor, The friction and lubrication of solids, 4th ed. (Clarendon Press, Oxford, 1986).

[16] J. Galambos, The asymptotic theory of extreme order statistics (Wiley, New York, 1978).

[17] V. M. Vinokur, M. C. Marchetti, and L.-W. Chen, Phys. Rev. Lett. 77, 1845 (1996).

[18] P. Sollich, F. Lequeux, P. Hébraud, and M. E. Cates, Phys. Rev. Lett. 78, 2020 (1997).

[19] J. P. Bouchaud, Preprint cond-mat/9910387; J. P. Bouchaud, and M. Mézard, J. Phys. A 30, 7997 (1997).

[20] A. Coniglio, and M. Nicodemi, J. Phys.: Condens. Matter 12, 6601 (2000).

\section{Figure captions}

FIG. 1. Sketch of the forced torsion oscillator immersed at a depth $L$ into a granular medium of glass beads. A single layer of glass beads is glued to the oscillator probe, and the effective radius is $R_{e}$. The container, filled with the granular material, is shaken vertically by a vibrator at the intensity of vibration, $\Gamma$, below the fluidization limit. The method provides a measure of the complex frequency response of the granular medium while the vibrator mimics "thermal" fluctuations. $1=$ suspension wires; $2=$ permanent magnet; $3=$ external coils;
$4=$ mirror; $5=$ probe; $6=$ vibrator.

FIG. 2. Oscillator frequency response, at $f_{p}=1 \mathrm{~Hz}$, as a function of the amplitude of the applied torque $T_{0}$, for different vibration intensities $\Gamma$, for $f_{s}=200 \mathrm{~Hz}$. (a) The loss factor, given by $\tan \left[\arg \left(G_{1}\right)\right]$, versus $T_{0}$. The position of the peak obtained at $\Gamma<2 \times 10^{-3}$ (i.e., with the vibration off) is denoted $T_{0}^{*}$. (b) The modulus $\left|G_{1}\right|$ versus $T_{0}$. The two extreme levels on the curve obtained at $\Gamma<2 \times 10^{-5}$ are denoted $G_{p}$ and $G_{j a m}$ respectively. For $T_{0} \ll T_{0}^{*}$, the response is independent of $T_{0}$, i.e., there is a linear regime, confirmed also by a negligible high harmonics signal (not shown) for all $\Gamma$.

FIG. 3. Oscillator frequency response as a function of the vibration intensity $\Gamma$, with $f_{s}=200 \mathrm{~Hz}$, for different forcing frequencies $f_{p}$ of the oscillator, at a given low torque amplitude $T_{0}=3.2 \times 10^{-5} \mathrm{~N} \mathrm{~m}$ selected in the linear regime, i.e., $T_{0} \ll T_{0}^{*}$. (a) The loss factor $\tan \left[\arg \left(G_{1}\right)\right]$, versus $\Gamma$. (b) The modulus $\left|G_{1}\right|$ versus $\Gamma$. For each $f_{p}$ a peak with a maximum at a vibration intensity $\Gamma^{*}$ can be seen. The data shown in Fig. 3 are collected by decreasing $\Gamma$, but no difference is observed in following runs if $\Gamma$ is successively increased, decreased and so on (see Fig. 4.).

FIG. 4. Similar to Fig. 3 , for $f_{p}=1 \mathrm{~Hz}$, but for $\Gamma$ successively decreased, increased, decreased and so on.

FIG. 5. Oscillator frequency response as a function of the forcing frequency $f_{p}$, for different vibration intensity $\Gamma$, with $f_{s}=200 \mathrm{~Hz}$, at a given torque amplitude $T_{0}=$ $3.2 \times 10^{-5} \mathrm{~N} \mathrm{~m}$. (a) The loss factor $\tan \left[\arg \left(G_{1}\right)\right]$, versus $f_{p}$. (b) The modulus $\left|G_{1}\right|$ versus $f_{p}$. One can see for each $\Gamma$ a peak with a maximum at a frequency $f_{p}^{*}$. The data shown are collected by decreasing $f_{p}$. A Debye peak of equation $C \omega_{p} \tau_{c} /\left(1+\omega_{p}^{2} \tau_{c}^{2}\right)$ with $C=1.9$ and $\tau_{c}=$ $R^{-1}=0.6$ is shown in (a). As a function of the frequency, the shape of the Debye peak is independent of the exact definition of the temperature-like parameter entering the rate $R$. The observed "jamming" peaks are much larger than the pure Debye peak, suggesting that the underlying dynamics is glassy in nature.

FIG. 6. The semilogarithmic Arrhenius-like plot reporting the forcing frequency $f_{p}$ versus $1 / \Gamma$ of "jamming" peaks similar to the ones in Figs. 3 and 5 (filled symbols from measurements vs. $\Gamma$; open symbols from measurements vs. $\left.f_{p}\right)$, for different vibration frequencies $f_{s}$. The data are fitted (dashed lines) by $2 \pi f_{p}=\nu_{0} \exp \left(-\Gamma_{j} / \Gamma\right)$, which gives $\nu_{0} \approx 70 \mathrm{~Hz}$ and $\Gamma_{j} \approx 0.014$ for $f_{s}=50 \mathrm{~Hz}$, $\nu_{0} \approx 66 \mathrm{~Hz}$ and $\Gamma_{j} \approx 0.042$ for $f_{s}=100 \mathrm{~Hz}$, and $\nu_{0} \approx 49 \mathrm{~Hz}$ and $\Gamma_{j} \approx 0.14$ for $f_{s}=200 \mathrm{~Hz}$.

FIG. 7. The forcing frequency $f_{p}$ versus the inverse of the empirical control parameter $1 / \tau$, i.e., versus $\omega_{s} / \Gamma^{n}$, with $n=1 / 2$. The data are fitted (plain and dashed lines) by $2 \pi f_{p}=\nu_{0} \exp \left(-\tau_{j} / \tau\right)$, which gives $\nu_{0} \approx 1336 \mathrm{~Hz}$ and $\tau_{j} \approx 1.3 \times 10^{-3} \mathrm{~s}$ for $f_{s}=50 \mathrm{~Hz}, \nu_{0} \approx 1465 \mathrm{~Hz}$ and $\tau_{j} \approx 1.2 \times 10^{-3} \mathrm{~s}$ for $f_{s}=100 \mathrm{~Hz}, \nu_{0} \approx 2698 \mathrm{~Hz}$ and $\tau_{j} \approx 1.2 \times 10^{-3} \mathrm{~s}$ for $f_{s}=200 \mathrm{~Hz}$. The straight lines have almost the same "slope" $\tau_{j}$. The average is $\left\langle\tau_{j}\right\rangle=1.26 \times$ 
$10^{-3} \mathrm{~s} . \nu_{0}$ is seen as a natural vibration frequency of the granular medium. Considering the present precision of the data, no clear relationship between $\nu_{0}$ and $f_{s}$ can be found, even though $\nu_{0}$ increases as $f_{s}$ increases.

FIG. 8. Similar to Fig. 7, but with $n=0.57$. The data are fitted (plain and dashed lines) by $2 \pi f_{p}=$ $\nu_{0} \exp \left(-\tau_{j} / \tau\right)$, which gives $\nu_{0} \approx 648 \mathrm{~Hz}$ and $\tau_{j} \approx 8.0 \times$ $10^{-4} \mathrm{~s}$ for $f_{s}=50 \mathrm{~Hz}, \nu_{0} \approx 683 \mathrm{~Hz}$ and $\tau_{j} \approx 7.8 \times 10^{-4} \mathrm{~s}$ for $f_{s}=100 \mathrm{~Hz}, \nu_{0} \approx 1019 \mathrm{~Hz}$ and $\tau_{j} \approx 8.7 \times 10^{-4} \mathrm{~s}$ for $f_{s}=200 \mathrm{~Hz}$. 


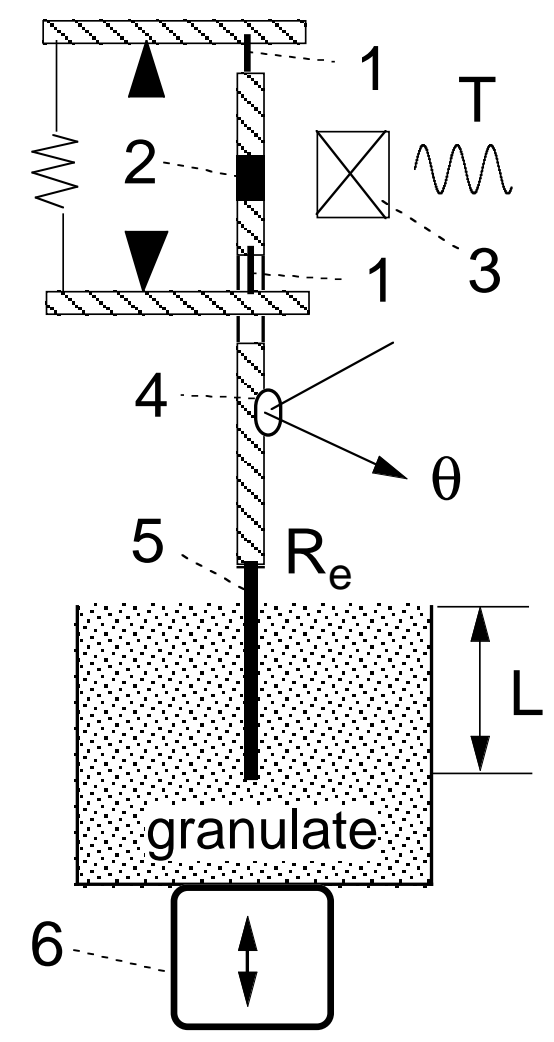




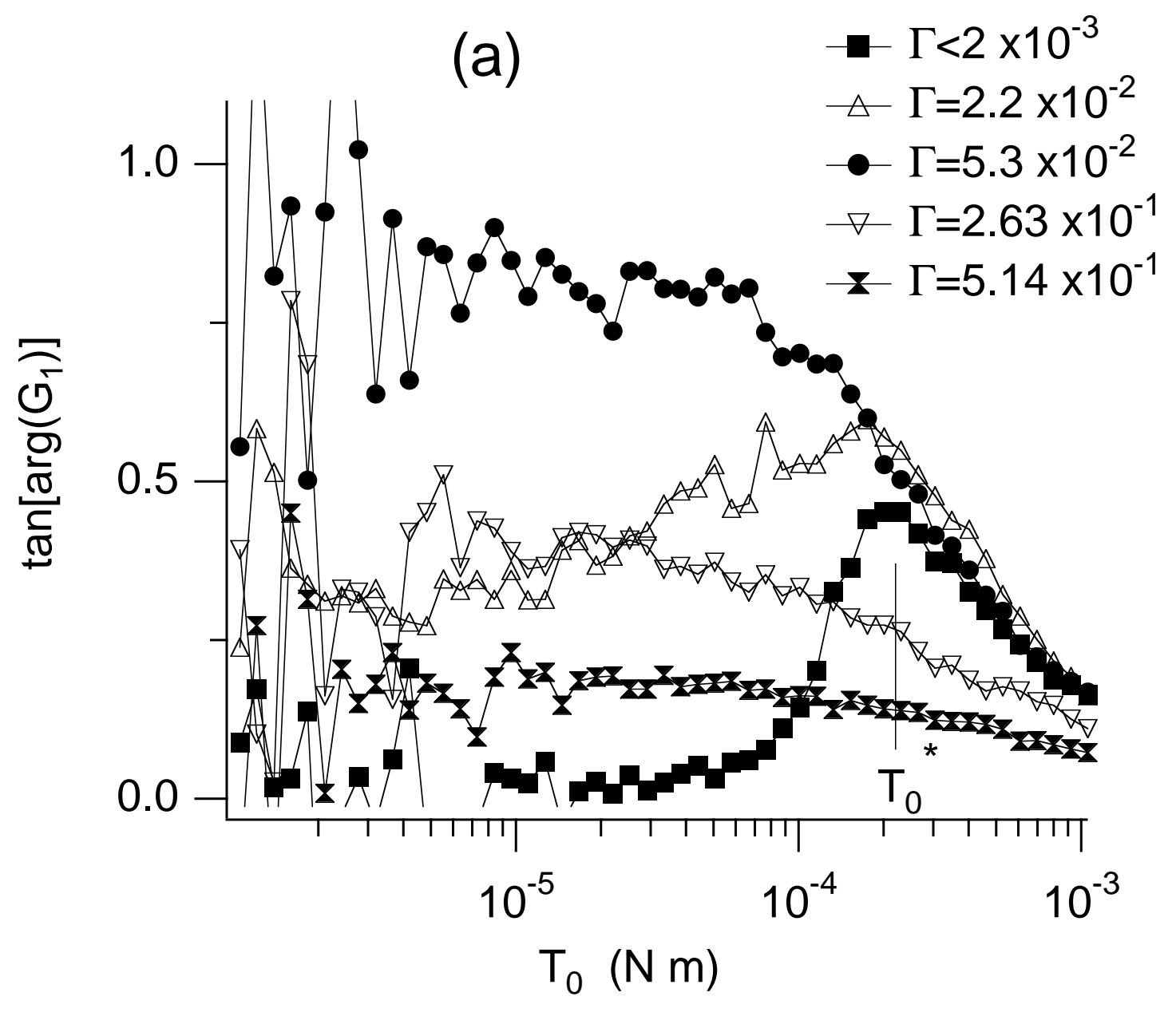

(b)

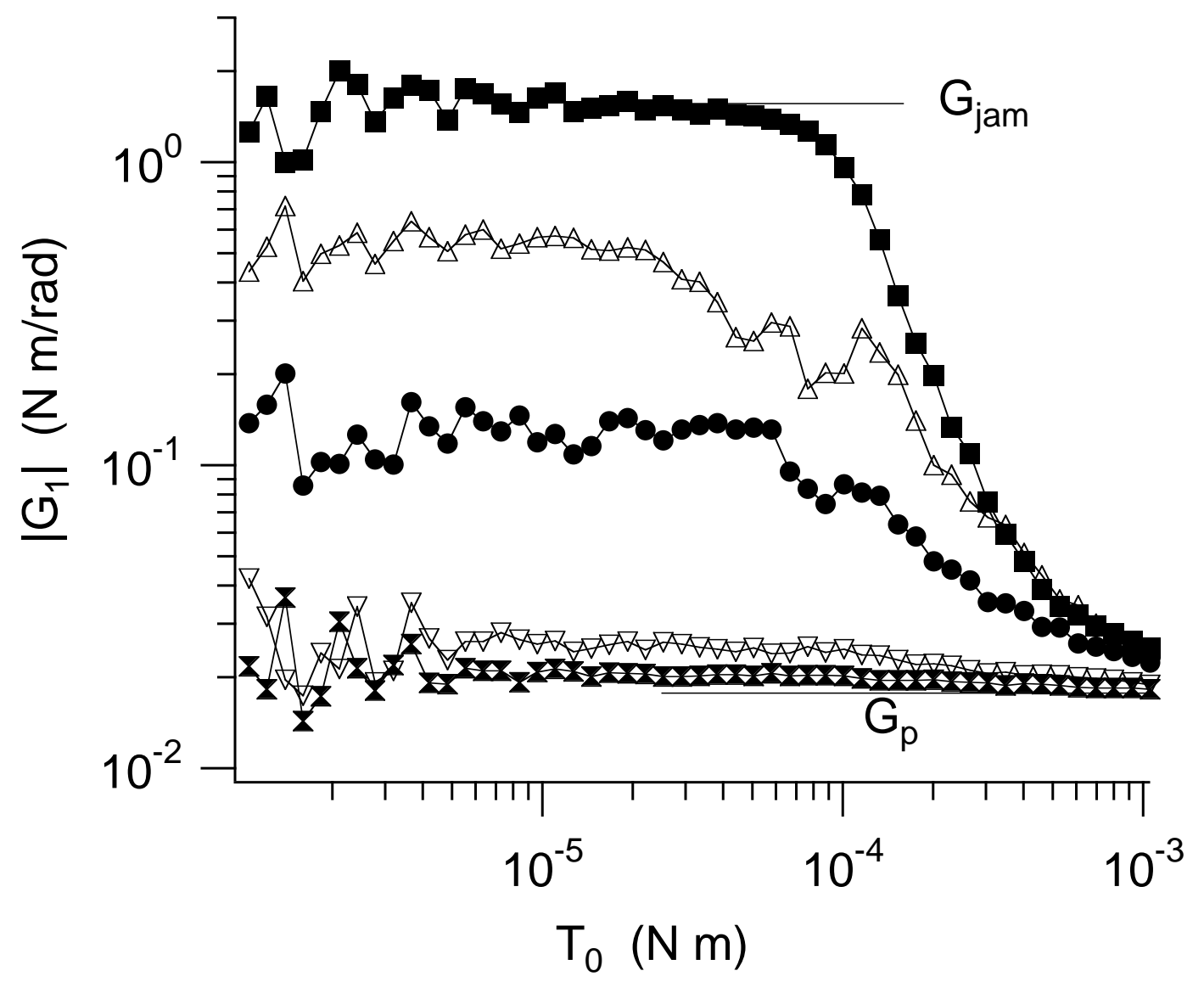



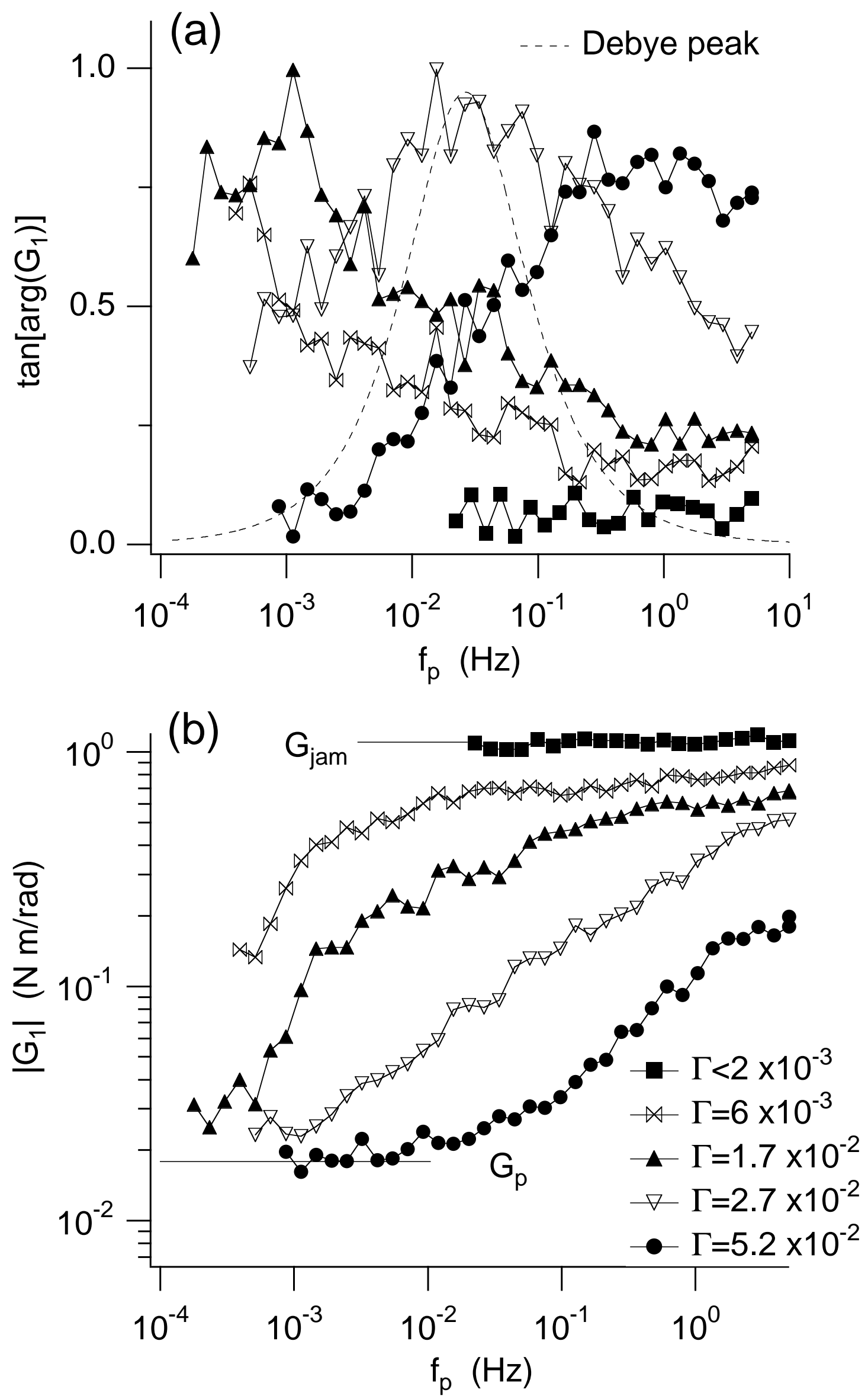


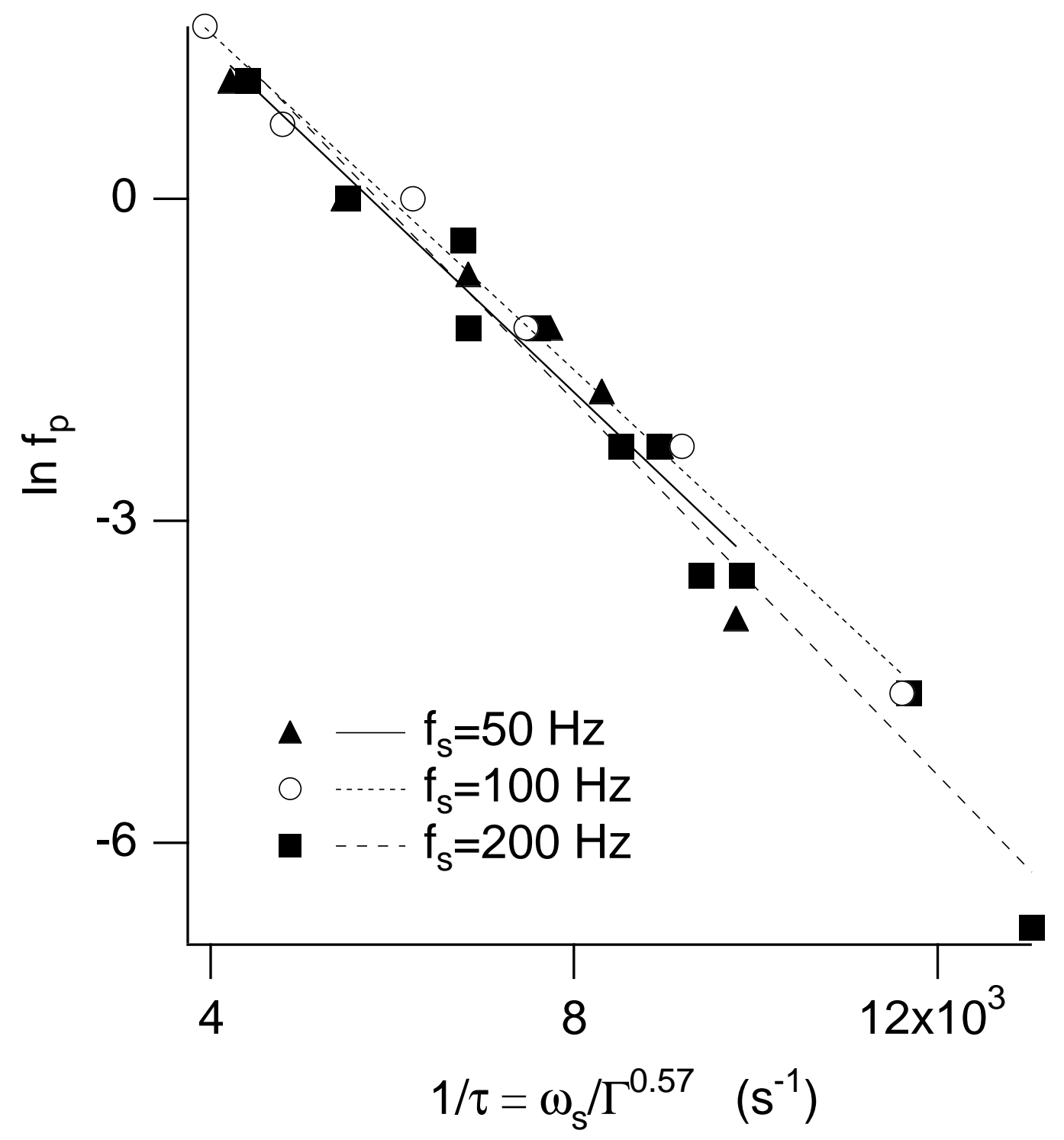



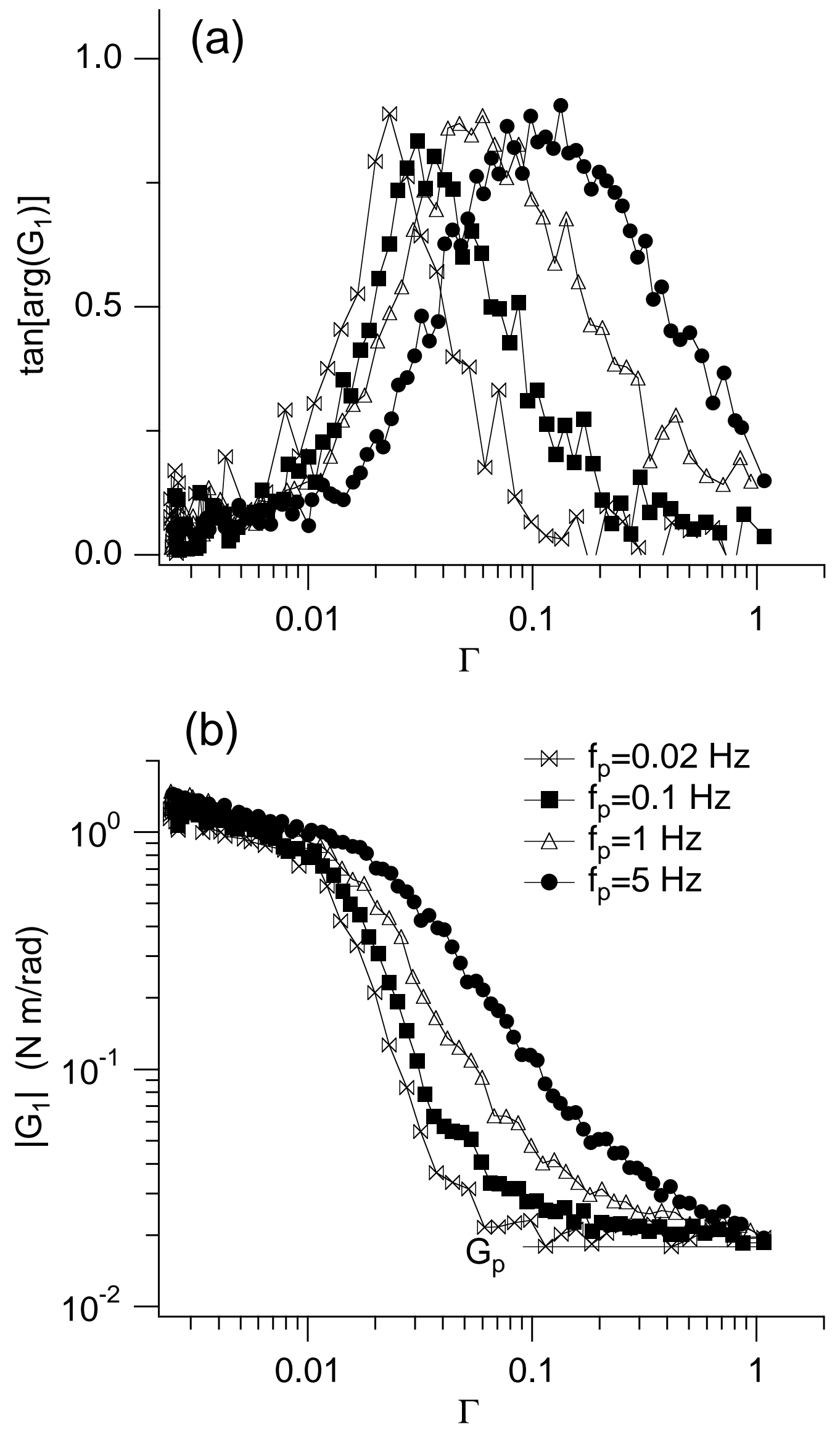


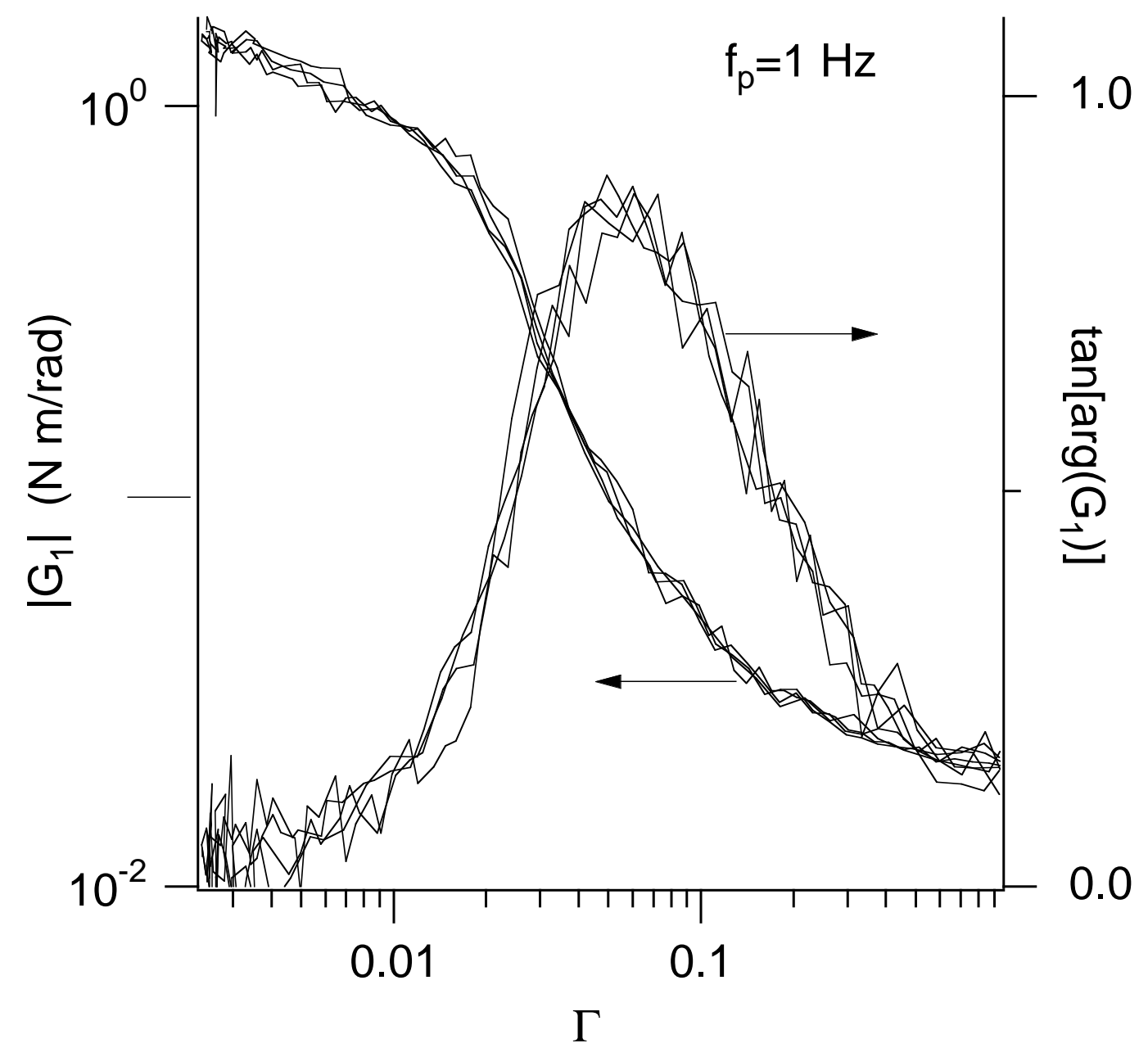




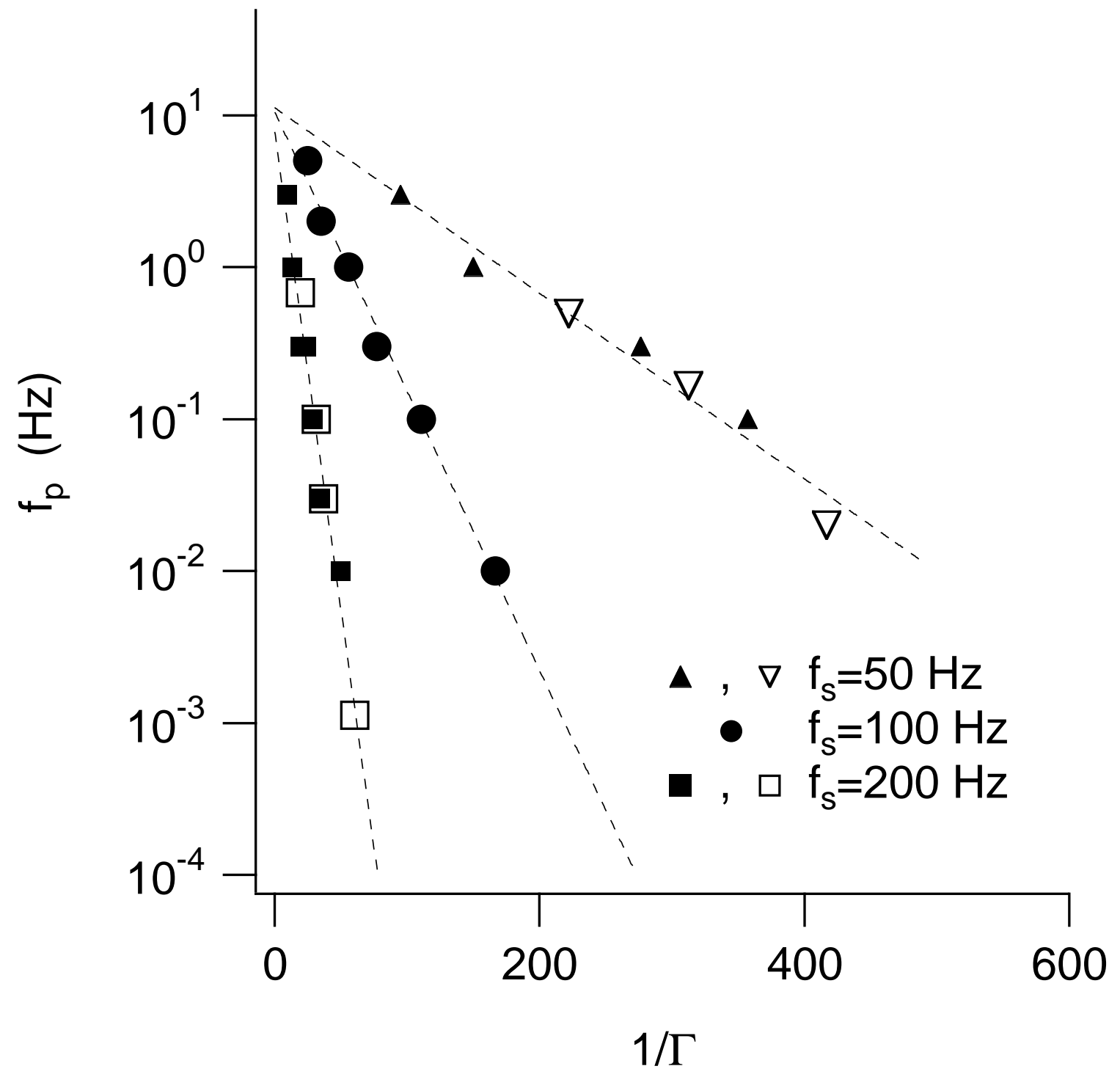




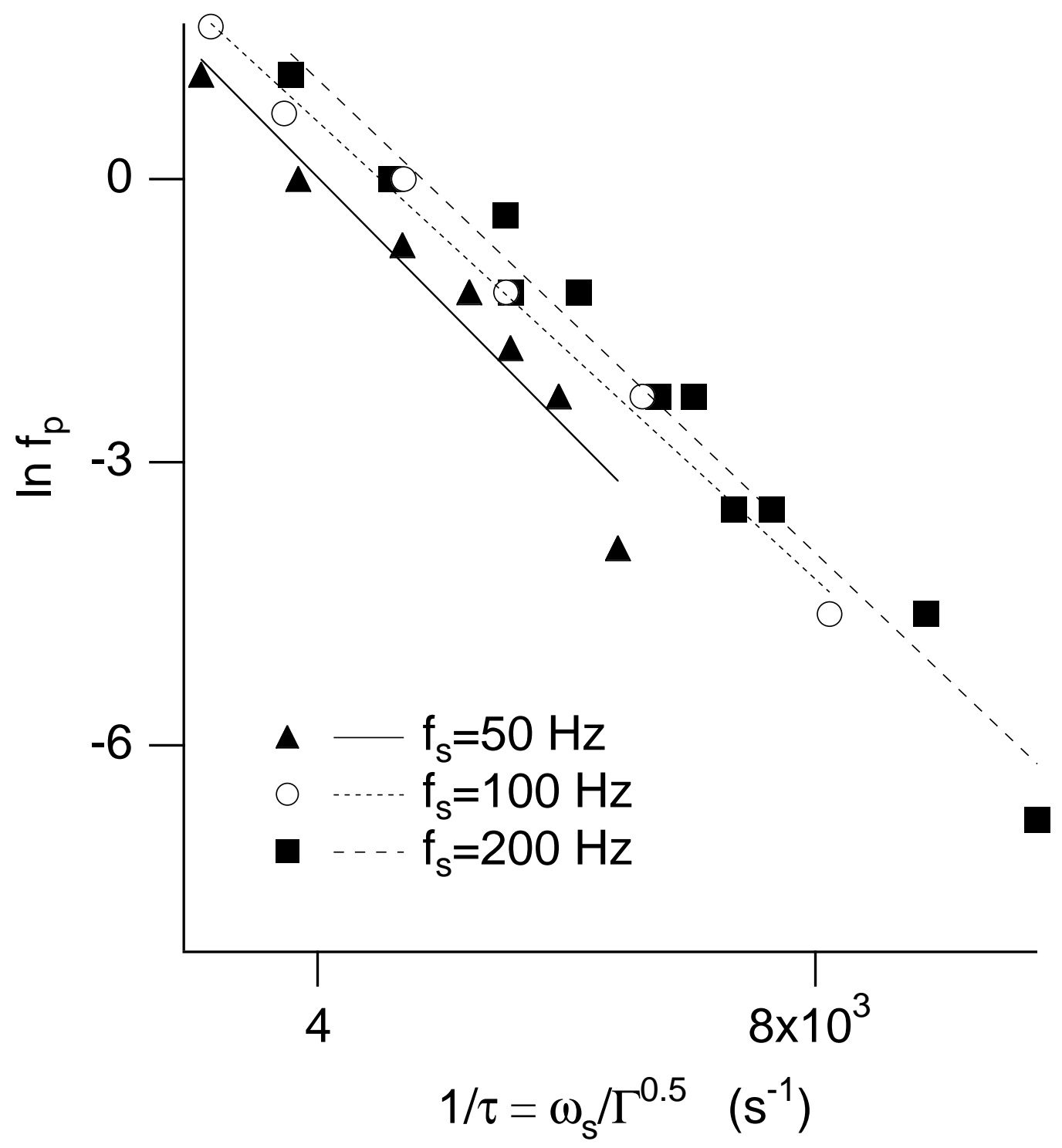

